\title{
Application and fabrication aspects of sub-micrometer-sized Josephson junctions
}

\author{
G. Oelsner ${ }^{1}$, U. Hübner ${ }^{1}$, S. Anders ${ }^{1}$, and E. Il'ichev ${ }^{1,2}$ \\ ${ }^{1}$ Leibniz Institute of Photonic Technology, P.O. Box 100239, Jena D-07702, Germany \\ E-mail: gregor.oelsner@leibniz-ipht.de \\ ${ }^{2}$ Novosibirsk State Technical University, 20 Karl Marx Ave., Novosibirsk 630092, Russia
}

Received March 2, 2017, published online May 25, 2017

\begin{abstract}
We present two possible methods for the fabrication of sub-micron sized Josephson junctions, namely the shadow-evaporation technique and the cross-type technology. Their importance for the field of modern superconducting technology is discussed. As examples we present measurement results of a two-qubit sample and a prototype of a microwave detector fabricated each by one of the described methods. We review potential applications of superconducting quantum circuits based on the developed methods.
\end{abstract}

PACS: 85.25.Cp Josephson devices;

85.25.Dq Superconducting quantum interference devices (SQUIDs).

Keywords: sub-micro Josephson junctions, fabrication, superconducting qubits.

\section{Introduction}

In spite of the fact that superconductivity was opened more than 100 years ago, this phenomenon still attracts attention of experts motivated mostly by its potential applications. By making use of fundamental laws of superconductivity - namely the quantization of magnetic flux inside of a closed loop [1,2] and the Josephson effect [3] - a nontrivial magnetic flux dynamic inside a superconducting structure can be realized. A simple example can be demonstrated by embedding a single or several Josephson junctions into a loop. In such an arrangement magnetic flux quanta can enter or leave the loop. Therefore, in this system and under certain conditions, two such flux states form the basis of a two-level quantum system [4] called a quantum bit (qubit). Similar qubits can be developed for the quantized number of Cooper pairs on a small superconducting island. There also a Josephson junction can allow individual pairs to enter or leave the island and thus couple different charge states [5]. Note that this device embedded into a superconducting loop results in a so-called interferometer-type charge qubit [6]. Qubits are successfully used for quantum-optic experiments [7-10] and strong research efforts are made towards quantum computing [11-17].

Using the current-biased superconducting loop with two Josephson junctions a sensitive magnetic field detector can be developed [18]. Such superconducting quantum interference device (DC SQUID) is biased slightly above the maximum of the superconducting current that can be fed to both junctions. The value of this maximal current, and therefore the voltage across the SQUID-structure, depends on the external magnetic field. In that way a reasonable large magnetic field-voltage transfer function is achieved.

On the other hand, a complete isolation from external fields is needed for digital electronics based on Josephson junctions. These so-called single flux quantum (SFQ) circuits are based on the flux quantization. The working principle can be demonstrated by making use of a simplified building block, namely a DC SQUID discussed above (see, for example [19]). The presence of an additional flux quantum in the loop will lower the total current at one and increase it at the other junction. If a tact-pulse is applied one junction will switch and therefore release the flux quantum. By that a controlled digital operation is achieved and by the use of many junctions the fast (hundreds of $\mathrm{GHz}$ ) electronic circuits can be fabricated [20].

All of these applications benefit from the fabrication of sub-micron sized Josephson junctions. In this article we discuss two well established methods for their fabrication and give examples of measurement results. The paper is organized as follows. In Sec. 2 we discuss the need of smallscale junctions with special focus on the application in the field of macroscopic quantum phenomena. The fabrication methods are explained in Sec. 3. Afterwards measurement results on a two-qubit sample and a microwave detector fabricated respectively with each method are shown in Sec. 4. Finally a conclusion summarizes the paper. 


\section{Motivations for sub-micron Josephson junctions}

The use of small-scale Josephson junction is preferable because of the different reasons that depend on the application. For example with a lowered parasitic capacitance the usable voltage swing of a SQUID is increased while at the same time both the energy resolution as well as the intrinsic flux noise take lower values [21].

To be competitive with the well established standard semiconductor technology, progress in the level of integration in SFQ electronics is required [22]. Small-scale Josephson junctions allow an increase of their densities on chip and thus are one step in this direction. The decrease in junctions size should be accompanied by an increase in the critical current density such that the value of critical current for the junctions remains. This requirement ensures the stability of the digital circuits.

While for the above mentioned applications sub-micron sized junctions are preferable, they are required for macroscopic quantum experiments. There the ratio between Josephson energy $E_{J}=I_{c} \Phi_{0} / 2 e$ and charging energy $E_{C}=2 e^{2} / C$ is a key parameter. Here, $I_{c}$ is the critical current and $C$ is the capacitance of the junction. Also the physical constants of electric charge $e$ of an electron and magnetic flux quantum $\Phi_{0}$ are used. In a simplified explanation the comparison of the Josephson and charging energy gives a relation of the energy of the quantum states and their coupling. Thus the ratio ranges from a value of 100 for flux [4] to 0.1 for charge qubits [5]. Note that in principle also the total energy of the states should have the right order of magnitude to be feasible for detection and manipulation. The required area $A$ of the Josephson junction is than found by

$$
A=2 e \sqrt{\frac{E_{J}}{E_{C}} \frac{1}{j_{c} c \Phi_{0}}}
$$

where $j_{c}$ is the critical current density and $c$ is the sheet capacitance of the junction. The latter takes a value of about $60 \mathrm{fF} / \mu \mathrm{m}^{2}$ for tunnel barriers formed by $\mathrm{AlO}_{x}$. With a reasonable current density of $200 \mathrm{~A} / \mathrm{cm}^{2}$ we find junction areas of $0.2 \mu \mathrm{m}^{2}$ for flux and of the order of $0.01 \mu \mathrm{m}^{2}$ for charge qubits. These values illustrate the need of submicron sized Josephson junctions. Note, even if the current density is lowered for charge qubits the size of the junctions still has roughly the same order of magnitude because of the weak square-root dependency.

\section{Fabrication}

\subsection{Shadow evaporation}

The fabrication process starts on 4"-Si-wafers which are prepared with wiring-, resonator- or coil-structures and alignment marks made from niobium. On such a wafer several chip layouts are arranged, see Fig. 1 with two ex-

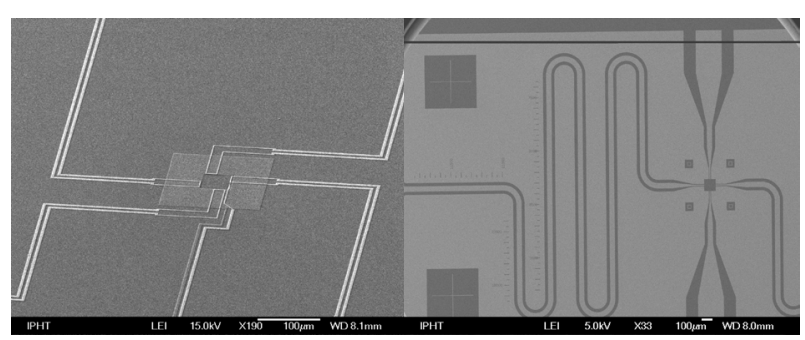

Fig. 1. SEM-images showing examples of $\mathrm{Nb}$-structures ready for the shadow-evaporation technique. On the left picture a qubit architecture should be placed inside of the coil. The latter forms a resonant circuit at frequency of several tens of megahertz with an external capacitance. A gigahertz frequency coplanar resonator is shown on the right image. On both designs additional lines for control fields are visible.

amples. Each chip has a size of $10 \times 10 \mathrm{~mm}$. After slicing of the wafers, the structures with small Josephson junctions are produced separately on the chips in a next manufacturing step. This independent fabrication enables more flexibility and adjustment of the parameters.

The qubit structures with small $\mathrm{Al} / \mathrm{AlO}_{x} / \mathrm{Al}$ - tunnel junctions are fabricated by using the so-called shadowevaporation technique [23] and by means of electron beam lithography. Here, a thin two-layer e-beam resist is exposed with the electron beam writer LION (Vistec Electron beam $\mathrm{GmbH}$ ) and developed to produce small and undercutted resist masks. The LION tool works with a beam energy of $20 \mathrm{keV}$ and a beam current of around $100 \mathrm{~mA}$. The two resists are ARP617 at the bottom and ARP6200 on the top with respective thicknesses of $300 \mathrm{~nm}$ and $120 \mathrm{~nm}$. Both are supplied by Allresist GmbH Berlin. After the e-beam exposure the resists are developed separately: first $60 \mathrm{~s}$ by using AR600-546 developer followed by $60 \mathrm{~s}$ in AR600-50 and finished by rinse in a isopropanol-bath.

The junctions are produced by an electron gun evaporation and oxidation of aluminium using a two-angle deposition-oxidation-deposition cycle on the prepared substrates (a)

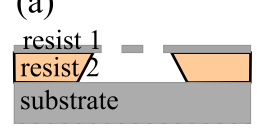

(b)

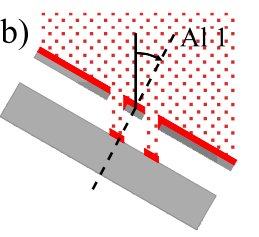

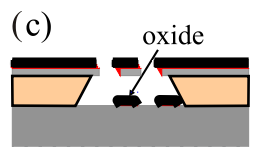

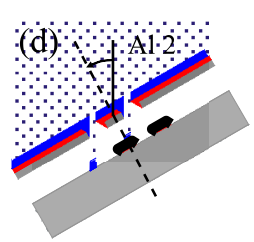

(e)

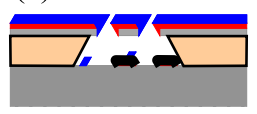

(f)

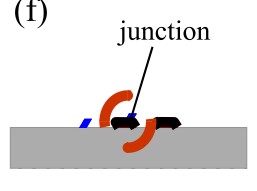

Fig. 2. (Color online) Schematic of the shadow-evaporation fabrication process as described in the main text. 


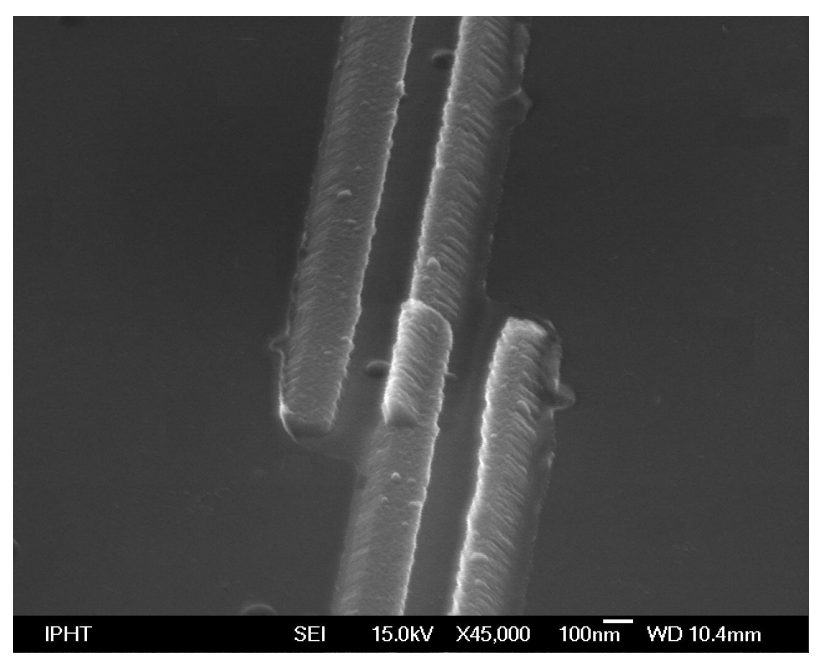

Fig. 3. Tilted SEM-picture of a typical $\mathrm{Al} / \mathrm{AlO}_{x} / \mathrm{Al}$-tunnel junction fabricated by the two-angle shadow-evaporation technique.

(see Fig. 2). First; a $50 \mathrm{~nm}$ thick $\mathrm{Al}$ film is evaporated and then oxidized for several minutes to form the tunnel barrier. Then the sample is tilted back to the opposite angle and the second $80 \mathrm{~nm}$ thick $\mathrm{Al}$ film is evaporated. The process is completely realized in a single vacuum cycle. Finally, the resist mask is removed using a solvent bath (AR 600-71) and the qubit structures with the tunnel junctions are obtained. Their typical sizes are about $200 \times 500 \mathrm{~nm}$ and the junctions have current densities in the order of $500 \mathrm{~A} / \mathrm{cm}$ typically. An example of a junction produced in that way is shown in Fig. 3.

\subsection{Cross-type technology}

Cross-type Josephson junctions are usually fabricated on wafer scale. Therefore, not only the junctions, but also control and microwave lines as well as readout structures are produced not individually on chips, but on the entire wafer at the same time.

The process starts by sputter depositing a $\mathrm{Nb}-\mathrm{AlO}_{x}-\mathrm{Nb}$ trilayer on a 4-inch silicon wafer. Lithography with an i-line waferstepper forms a resist mask in the shape of a narrow stripe. The trilayer is structured by reactive ion beam etching and sputter etching. The sidewalls of the trilayer stripe are passivated by anodic oxidation. Next, $\mathrm{SiO}$ is thermally evaporated, followed by lift-off in ultrasonically agitated acetone. Now the wafer is coated with a $\mathrm{Nb}$ layer. This layer is structured into a stripe that is perpendicular to the trilayer stripe. Etching down to the Al barrier ensures that the junction is formed at the overlap of the two stripes. Compared to alternative fabrication processes for $\mathrm{Nb}-\mathrm{AlO}_{x}-\mathrm{Nb}$-based Josephson junctions, the parasitic capacitance of the crosstype junctions is minimized. The cross-type fabrication pro-

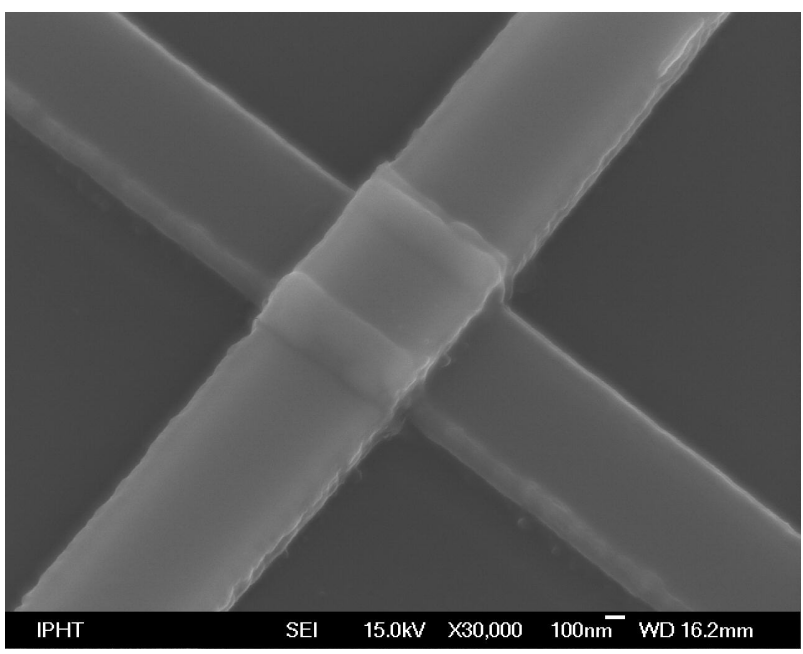

Fig. 4. SEM image of a cross-type Josephson junction with a size of nominally $0.8 \times 0.8 \mu \mathrm{m}$.

cess has been described in detail, together with the characterization of the junctions, in Ref. 24.

Figure 4 shows an example of a cross-type Josephson junction. Junctions are formed at each position where the two layers overlap. Either layer can be used for control and readout lines. An example of two qubits is shown in Fig. 5. Each qubit has a quadratic geometry with Josephson junctions in their corners. The qubits are placed in the current antinode of a coplanar waveguide resonator. Additionally, the qubits share one arm with the central conducting line resonator. For the application of $\mathrm{dc}$ and microwave fields, two external control lines are structured into the trilayer. The functionality of these qubits has not yet been experimentally verified.

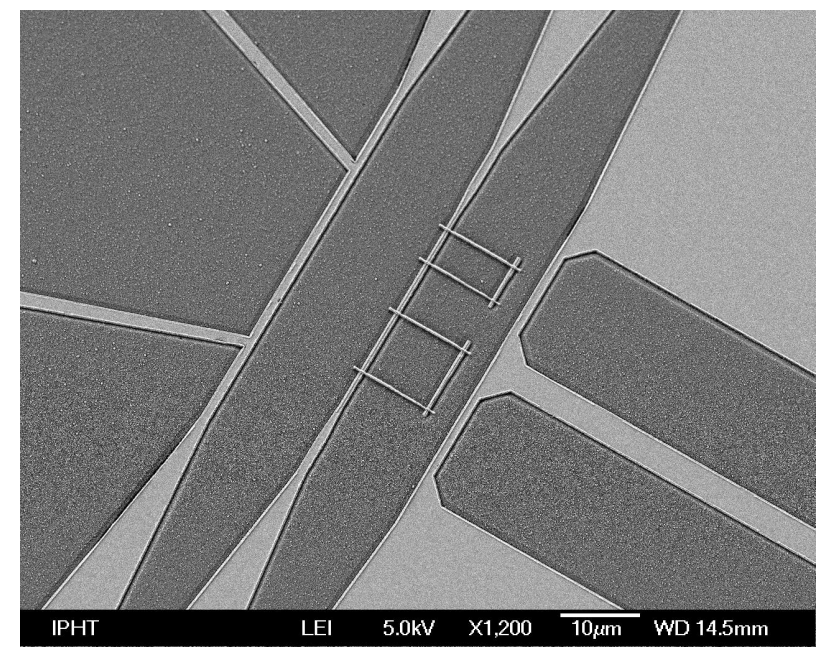

Fig. 5. SEM image of an example of a two-qubit sample fabricated by the cross-type technology. 


\section{Application examples}

\subsection{Two-qubit sample}

The shadow-evaporation technique is regularly used for the fabrication of macroscopic quantum systems. In Fig. 6 a SEM-image of two flux qubits fabricated to the center of a coplanar waveguide resonator is shown. The qubit-loops have sizes of $2.5 \times 5 \mu \mathrm{m}$ and $5 \times 5 \mu \mathrm{m}$. Each includes three Josephson junctions. They are designed to have sizes of $150 \times 750 \mathrm{~nm}$ for the two big junctions and $150 \times 500 \mathrm{~nm}$ for the small. An oxidation time of $6.5 \mathrm{~min}$ was used for an aimed critical current density of $j_{c}=200 \mathrm{~A} / \mathrm{cm}^{2}$.

For the experiment the sample is mounted at the base stage of a dilution refrigerator at a nominal temperature of about $20 \mathrm{mK}$. Mu-metal and superconducting shields decrease the influence of external field noise. Attenuators at the resonator input line and a circulator at its output isolate the sample from room temperature noise. An external bias coil for applying magnetic field is wired over low-pass filters at different temperature stages.

The fundamental half-wavelength mode of the resonator at $v_{r}=2.5 \mathrm{GHz}$ produces a current antinode at the qubits position. The quality factor of the resonator has a value of $Q=2.2 \cdot 10^{4}$ at $20 \mathrm{mK}$. The qubit parameters, namely the minimal level splittings $h \Delta_{k}$, the persistent current $I_{p k}$, and the coupling constant to the resonator $h g_{k}=M_{k} I_{p k} I_{r}$, can all be extracted from the measurements of the resonator transmission [25-27] and [28]. The subscript $k$ is used to identify the values of each qubit were " 1 " and " 2 " are used, respectively, for the large and small qubit. The values for the mutual inductance $M_{k}$ between the qubits and the resonator are estimated from their geometry to be 0.8 and $1.3 \mathrm{pH}$ for the small and big loop, respectively. The zero point current

$$
I_{r}=\sqrt{\frac{h \mathrm{v}_{r}}{L_{r}}}
$$

of the resonator follows from its inductance $L_{r} \approx 13 \mathrm{nH}$ and resonant frequency $[29,30]$. The transmitted phase

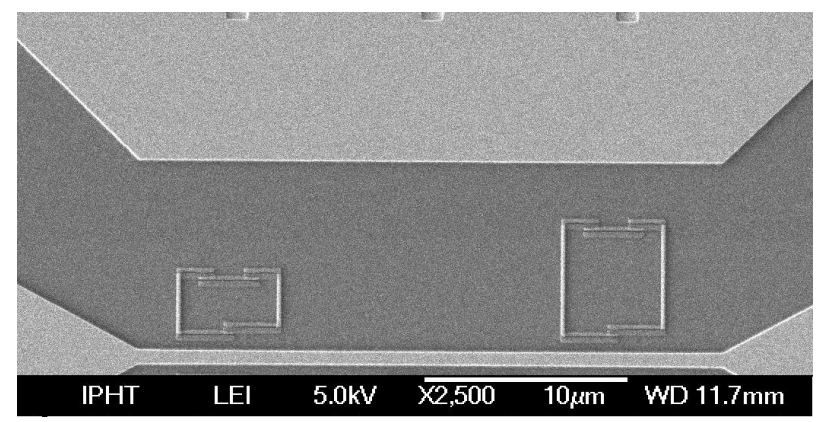

Fig. 6. SEM picture of a qubit sample fabricated by shadow evaporation. Two qubit loops are fabricated at the center of a coplanar waveguide resonator.

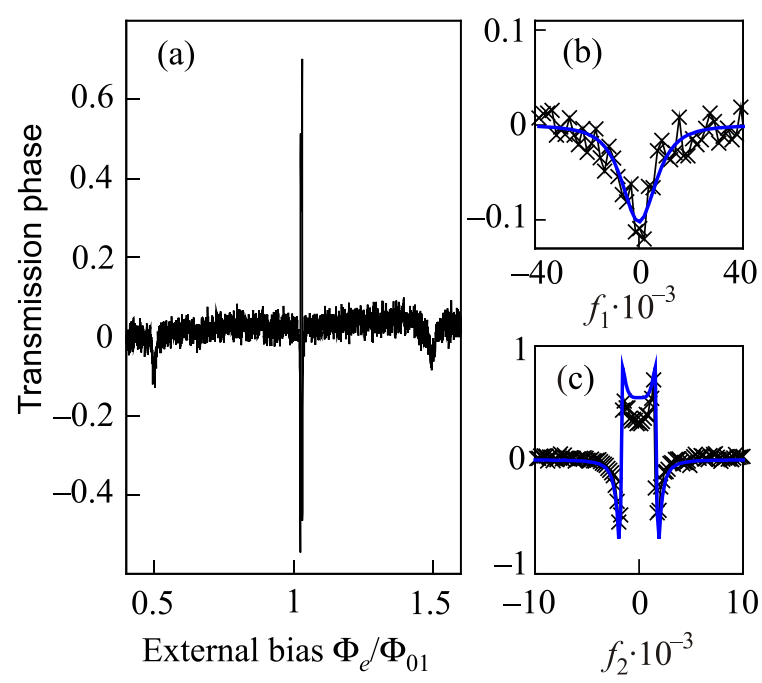

Fig. 7. (Color online) Phase response of the two-qubit-resonator sample measured at the resonant frequency $\omega_{p}=\omega_{r}$ in dependency of the external magnetic flux. In (a) the external magnetic flux is varied over a wide range. The results in (b) and (c) are found close to the degeneracy points of the two qubits.

over the externally applied flux $\Phi_{e}$ reveals characteristic dependencies for measurements on qubits. They are demonstrated in Fig. 7(a). Two dip structures together with a strong phase jump in between are experimentally observed. The dips can be associated to the bigger qubit, because they have half of the period in magnetic flux compared to the phase jump. The $x$-axes is scaled in units of flux quanta $\Phi_{01}$ in the big loop. Therefore, the dips are observed at 0.5 and 1.5 quanta. Because of the half area of the small qubit its degeneracy point is roughly found close to one flux quanta in the big qubit. The applied magnetic field leads to an energy bias $h \varepsilon_{k}=2 I_{p k}\left(\Phi_{e}-0.5 \Phi_{0 k}\right)$ that contributes to the qubits level splitting $v_{q k}=\sqrt{\varepsilon_{k}^{2}+\Delta_{k}^{2}}$.

The phase response of both qubits for a resonator probed at its resonant frequency can be calculated from the transmission coefficient [30,31]

$$
t=-\frac{i \kappa\left[\mathrm{v}_{q k}-\mathrm{v}_{p}-i \Gamma_{k}\right]}{i \kappa\left[\mathrm{v}_{q k}-\mathrm{v}_{p}-i \Gamma_{k}\right]+2 g_{\Delta k}^{2}} .
$$

Here $2 \pi \kappa$ is the lossrate of the resonator, $v_{p}$ is the probing frequency, $2 \pi \Gamma$ is the qubit's decoherence rate, and $g_{\Delta k}=g_{k} \Delta_{k} / v_{q k}$. Also it is assumed, that each qubit stays in the ground state during the measurement. This is justified for big detunings $v_{q k}-v_{p} \gg g_{k}$ or small probing amplitudes.

The dip structure of the bigger qubit indicates that its gap $\Delta_{1}$ is larger than the probing frequency $\omega_{p}$. This allows to assume large detunings and set $v_{q k}-v_{p} \gg \Gamma_{1}$ so 
that the transmission phase can be calculated from real and imaginary part of (3) as

$$
\varphi=\arctan \frac{\mathfrak{I}(t)}{\mathfrak{R}(t)}=\arctan \frac{2 h g_{1}^{2} \Delta_{1}^{2}}{\kappa v_{q 1}^{2}\left(v_{q 1}-v_{p}\right)} .
$$

With the definitions of $g_{1}, \varepsilon_{1}$, and the quality $Q=v_{r} / \kappa$ only $\Delta_{1}$ and $I_{p 1}$ remain as fitting parameters

$$
\varphi=\arctan \left(\frac{M^{2} Q}{h L_{r}} \frac{2 I_{p 1}^{2} \Delta_{1}^{2}}{v_{q 1}^{2}\left(v_{q 1}-v_{p}\right)}\right) .
$$

Note, the above equation can be interpreted as the ground state curvature [18], if the probing (and thus the resonator) frequency is small compared to the one of the qubit

$$
-\frac{1}{2} \frac{d^{2} v_{q}}{d \Phi_{e}^{2}}=\frac{2 I_{p 1}^{2} \Delta_{1}^{2}}{v_{q 1}^{3}} .
$$

A fit of the transmission phase of one of the qubit dips with (5) as shown Fig. 7(b) reveals the parameters of the big qubit. They are listed together with the ones for the small qubit in Tabl. 1. The latter are found by an additional

Table 1. Experimentally determined parameters of the qubits

\begin{tabular}{c|c|c}
\hline \hline & Big qubit & Small qubit \\
\hline \hline$\Delta, \mathrm{GHz}$ & 8.9 & 1.7 \\
$I_{p}, \mathrm{nA}$ & 140 & 180 \\
$g, \mathrm{MHz}$ & 3.1 & 2.4 \\
\hline \hline
\end{tabular}

\section{two tone spectroscopy as in [26].}

With these values the response of the resonant qubit can be directly calculated from (3). The additional parameter for the decoherence rate $2 \pi \Gamma$ was set to $50 \mathrm{MHz}$ for the calculated blue line in Fig. 7(c). Also in the resonant regime a good correspondence between experiment and theory is achieved.

The quite different parameters of the two qubits may at first be surprising. They have similar junctions geometries and are fabricated quite close to each other. Still, the parameters strongly (exponentially) depend on the ratio $\alpha$ of the small to the two big junctions in a loop. Note, that when for example starting in the lower Aluminium layer taking one turn over three Josephson junctions would end in the upper layer. Thus in the overlapping area between these two layers on one side of the qubit loop another large Josephson junction is needed to end in the starting layer. This additional large junction can be included by changing the ratio $\alpha$ to an effective value. Because the overlapping areas on the left and right arms differ by a factor of two for the two qubits, different ef- fective $\alpha$ and therewith also a large deviation of the qubit gaps can be expected.

To conclude, the reproducibility of the technology is best tested for equal layouts of the qubit structures. Experimental examples are found by the frequency division multiplexing readout of seven qubits [33] or a quantummetamaterial consisting of 20 qubits [34]. In the former experiment the mean gap and persistent current were respectively found to be $5.6 \mathrm{GHz}$ and $128 \mathrm{nA}$ with a standard deviation of $0.8 \mathrm{GHz}$ and $5 \mathrm{nA}$ for five of the qubits. The other two fabricated at the side of the chip were about $30 \%$ off. In the second mentioned experiment three collective modes with two times four and one time eight qubits was demonstrated. Also here the spread in the persistent current $74 \pm 2 \mathrm{nA}$ is small, while a large spread in the qubit gaps hindered the collective response of all 20 qubits.

\subsection{Microwave detector}

A microwave signal applied to $\lambda / 4$-superconducting resonator produces a standing wave with a current amplitude at the point where the resonator is shunted to ground. Especially for a single photon this current is of the size of the zero-point current (2) and of the order of tens of nanoampere for a frequency of the order of $\mathrm{GHz}$ and a coplanar waveguide resonator. A current biased underdamped Josepshon junction (CBJJ) can be used for the destructive detection of this amplitude. Even a similar realization as photon counter using resonant transfer of energy was proposed [35].

We used the cross-type technology to fabricate a prototype of such a detector and characterized its properties

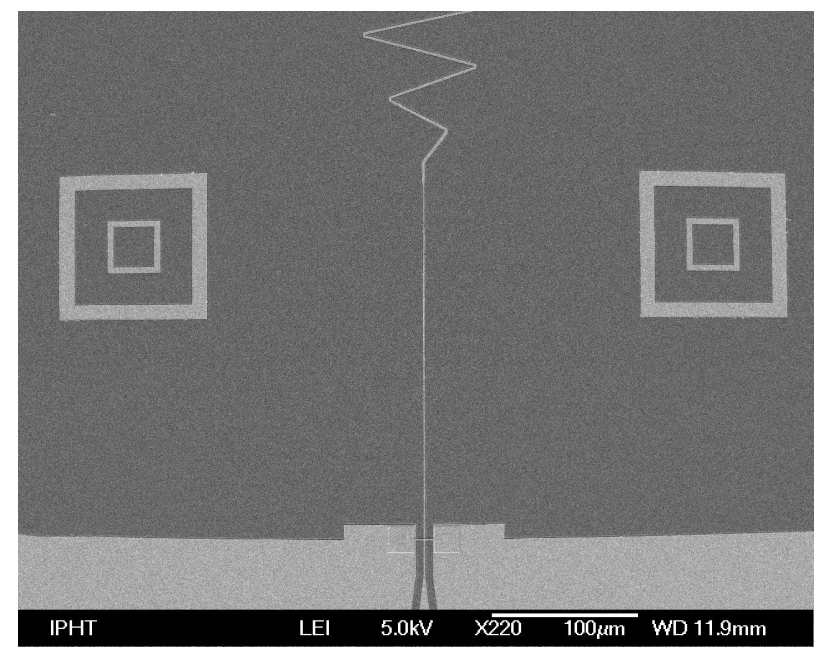

Fig. 8. SEM image of the detector sample close to the junctions position. At the bottom the end of the coplanar line is visible. It continues as thin bias line for the Josephson junction to the top of the figure. The second layer is visible as barbell structure shunting the resonator over one small and two large Josephson junctions. The dc-current line is closed via the mass plates of the resonator. 


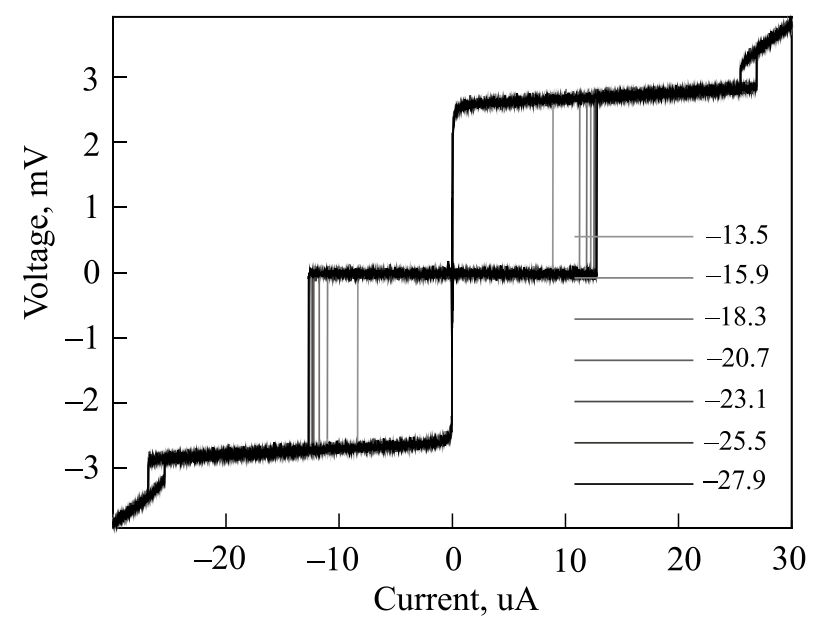

Fig. 9. Measured voltage-current characteristics of the Josephson junction in dependency of the applied microwave power. The legend shows its values in $\mathrm{dBm}$ at the cryostat head.

[36]. It consists of a Josephson junction incorporated into a $\lambda / 4$ coplanar waveguide resonator at it's connection to the ground plane (see Fig. 8). An additional transmission line for the application of microwave and the characterization of the resonator is capacitively coupled at the other end of the cavity. Its length is chosen to give a resonant frequency of $2.5 \mathrm{GHz}$.

For a first characterization the voltage-current characteristic of the junction is measured at different microwave input powers at the resonant frequency. The results are shown in Fig. 9. As expected a typical hysteretic curve is observed, where the supercurrent can be enlarged to a maximal value of $I_{c}$ before the junction switches to a finite voltage state. This gap voltage has a value about $2.6 \mathrm{mV}$ for the $\mathrm{Nb}$-technology, and thus is detectable without additional amplifiers. The switching current $I_{S}$ is shifted to lower values with increasing microwave power. That can be explained with the ac-current amplitude in the resonator added to the dc bias current.

The critical current $I_{c}$ is given by the junction size and thickness of the isolating barrier. But because the switching can be explained by an excitation out of a potential well $[9,11]$ he measured switching current $I_{S}$ is a statistical value and usually below $I_{c}$. External noise and the temperature can influence not only to its mean value but also to its distribution width. Quantum tunneling out of the potential well additionally limits the achievable switching current value and sets the lower limit for the amount of switching-current distribution when decreasing the temperature. Still standard deviations of the switching current well below $10 \mathrm{nA}$ are possible for optimized junction designs [39]. Note, that this would be sufficient for detection of single photons inside of the resonator.

For the presented detector it is therefore necessary to compare the width of the switching currents with the shift of

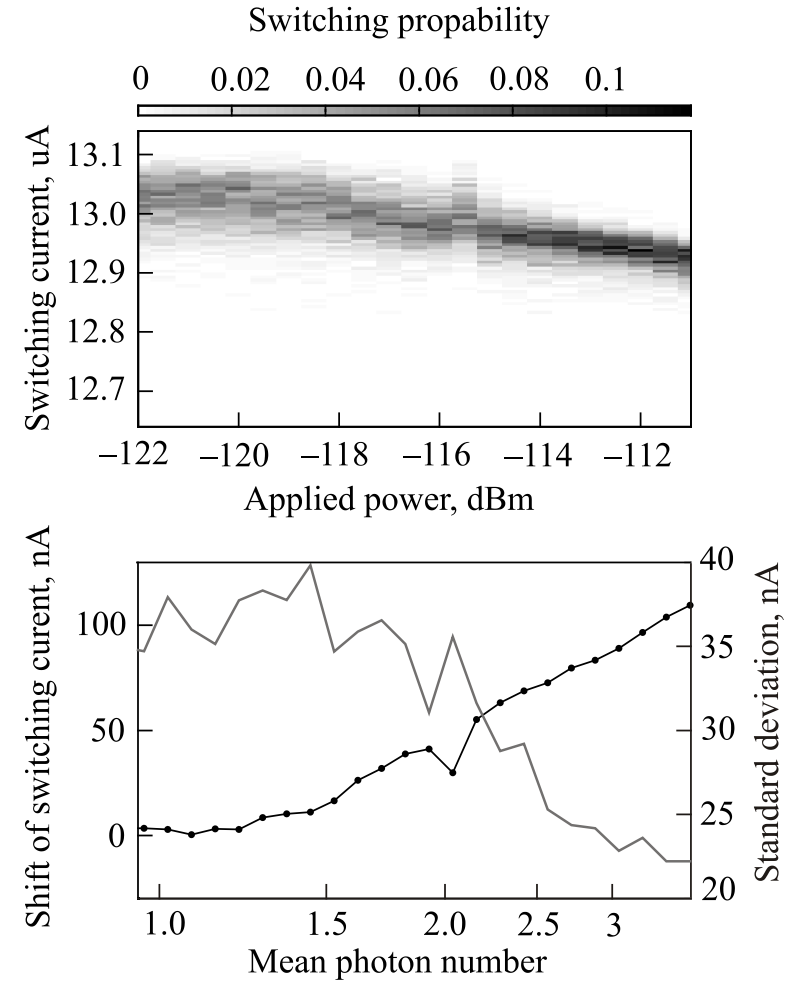

Fig. 10. The upper plot shows histograms of the switching current for different applied microwave powers. The distribution on the current is larger for smaller powers and the mean of the switching current approaches the undisturbed value. The extracted standard deviation and mean of the switching current is extracted and plotted over the recalculated photon number on the lower picture.

its mean value by the applied microwave signal. Corresponding measurement results at a frequency of about 2.48 GHz are shown in Fig. 10

There the histograms are shifted away from the undisturbed switching current $I_{s 0}$ to lower values with increasing applied microwave power. The reason is the increase of the current amplitude inside of the cavity. Thus a lower dc-bias current is needed to in sum reach the switching value. Also we note that the width of the distribution is lowered with increasing applied power.

For better visibility the shift of the switching current $I_{s 0}-I_{s}$ and its standard deviation $\sigma$ are shown in the lower plot of Fig. 10. A sensitivity of such detector may be defined as the shift of the switching current divided to the standard deviation. To achieve a normalization to one it can be extended to

$$
S=\frac{I_{s 0}-I_{s}}{I_{s 0}-I_{s}+\sigma} .
$$

A value of 0.5 is achieved were the curves in the lower plot of Fig. 10 cross. That is found at approximately two mean photons inside the cavity. For higher microwave powers (7) it reaches values close to one. Thus, after an calibration the applied microwave power can be inferred 
from the measurement of the mean switching current. Note, the increase of the bias current also will enlarge the Josephson inductance of the junction. Therewith the resonator frequency will shift to lower values making the sensitivity frequency dependent. For even higher detunings already a value of 0.5 of sensitivity at a single photon level is achieved for the discussed device [36].

With optimizations in the input coupling, the quality factor of the resonator, and especially of the junction parameters the discussed detector theoretically promises an increase in sensitivity to values close to one [40] and has prospects to be used as a single-photon detector.

\section{Conclusion}

As discussed above, the fabrication of small-scale Josephson junctions is a key technology. Applications that benefit from smaller junctions involve for example SQUIDmagnetometry or superconducting digital electronics. Most important modern fields as quantum computing or quantum optics on such macroscopic superconducting systems require fundamentally sub-micron-sized junctions. Two well established processes are available, namely the shadowevaporation technique and the cross-type technology. While the first mentioned is used for a flexible fabrication of junction structures on pre-processed chips the second allows a wafer scale fabrication. Also possible applications may benefit from the higher gap voltage achieved for the $\mathrm{Nb}$ process. As demonstrated on the experimental results both technologies have advantages in there respective applications namely reproducible production of quantum-bits and new designed architectures were control and readout are fabricated together with the Josephson junction.

\section{Acknowledgments}

G.O. thanks O. Astafiev, S.N. Shevchenko, C.K. Andersen, and K. Mølmer for the productive collaboration and helpful discussions. E.I. acknowledges partial support from the Russian Ministry of Education and Science, within the framework of State Assignment 3.8051.2017.

1. R. Doll and M. Näbauer, Phys. Rev. Lett. 7, 51 (1961).

2. B. Deaver and W. Fairbank, Phys. Rev. Lett. 7, 43 (1961).

3. B. Josephson, Phys. Lett. 1, 251 (1962).

4. J.E. Mooij, T.P. Orlando, L. Levitov, L. Tian, C.H. van der Wal, and S. Lloyd, Science 285, 1036 (1999).

5. Y. Nakamura, Y.A. Pashkin, and J.S. Tsai, Nature 398, 786 (1999).

6. D. Born, V.I. Shnyrkov, W. Krech, T. Wagner, E. Il'ichev, M. Grajcar, U. Hübner, and H.-G. Meyer, Phys. Rev. B 70, 180501(R) (2004).

7. A. Wallraff, D.I. Schuster, A. Blais, L. Frunzio, R.-S. Huang, J. Majer, S. Kumar, S.M. Girvin, and R.J. Schoelkopf, Nature 431, 162 (2004).
8. O. Astafiev, K. Inomata, A.O. Niskanen, T. Yamamoto, Y.A. Pashkin, Y. Nakamura, and J.S. Tsai, Nature 449, 588 (2007).

9. M.A. Sillanpää, J. Li, K. Cicak, F. Altomare, J.I. Park, R.W. Simmonds, G.S. Paraoanu, and P.J. Hakonen, Phys. Rev. Lett. 103, 193601 (2009).

10. A.A. Abdumalikov, O. Astafiev, A.M. Zagoskin, Y.A. Pashkin, Y. Nakamura, and J.S. Tsai, Phys. Rev. Lett. 104, 193601 (2010).

11. M. Grajcar, A. Izmalkov, and E. Il'ichev, Phys. Rev. B 71, 144501 (2005).

12. A. Izmalkov, M. Grajcar, S.H.W. v. d. Ploeg, U. Hübner, E. Il'ichev, H.-G. Meyer, and A.M. Zagoskin, Europhys. Lett. 76, 533 (2006).

13. J.H. Plantenberg, P.C. de Groot, C.J.P.M. Harmans, and J.E. Mooij, Nature 447, 836 (2007).

14. J. Koch, T. Yu, J. Gambetta, A. Houck, D. Schuster, J. Majer, A. Blais, M. Devoret, S. Girvin, and R. Schoelkopf, Phys. Rev. A 76, 042319 (2007).

15. L. DiCarlo, J.M. Chow, J.M. Gambetta, L.S. Bishop, B.R. Johnson, D.I. Schuster, J. Majer, A. Blais, L. Frunzio, S.M. Girvin, and R.J. Schoelkopf, Nature 460, 240 (2009).

16. A. Fedorov, L. Steffen, M. Baur, M.P. da Silva, and A. Wallraff, Nature 481, 170 (2011).

17. F. Yan, S. Gustavsson, A. Kamal, J. Birenbaum, A.P. Sears, D. Hover, T.J. Gudmundsen, D. Rosenberg, G. Samach, S.Weber, F. Yan, S. Gustavsson, A. Kamal, J. Birenbaum, A.P. Sears, D. Hover, D. Rosenberg, G. Samach, T.J. Gudmundsen, J.L. Yoder, T.P. Orlando, J. Clarke, A.J. Kerman, and W.D. Oliver, Nature Commun. 7, 12964 (2016).

18. R.C. Jaklevic, J. Lambe, A.H. Silver, and J.E. Mercereau, Phys. Rev. Lett. 12, 159 (1964).

19. J. Ren and V.K. Semenov, IEEE Transactions Appl. Superconduct. 21, 780 (2011).

20. W. Chen, A.V. Rylyakov, V. Patel, J.E. Lukens, and K.K. Likharev, Appl. Phys. Lett. 73, 2817 (1998).

21. M. Schmelz, R. Stolz, V. Zakosarenko, S. Anders, L. Fritzsch, M. Schubert, and H.-G. Meyer, Supercond. Sci. Technol. 24, 015005 (2011).

22. S.K. Tolpygo, Fiz. Nizk. Temp. 42, 463 (2016) [Low Temp. Phys. 42, 361 (2016)].

23. J. Niemeyer, PTB-Mitt. 84, 251 (1974).

24. S. Anders, M. Schmelz, L. Fritzsch, R. Stolz, V. Zakosarenko, T. Schönau, and H.-G. Meyer, Supercond. Sci. Technol. 22, 064012 (2009).

25. E. Il'ichev, T. Wagner, L. Fritzsch, J. Kunert, V. Schultze, T. May, H.E. Hoenig, H.G. Meyer, M. Grajcar, D. Born, W. Krech, M.V. Fistul, and A.M. Zagoskin, Appl. Phys. Lett. 80, 4184 (2002).

26. G. Oelsner, S.H. W. van der Ploeg, P. Macha, U. Hübner, D. Born, S. Anders, E. Il'ichev, H.-G. Meyer, M. Grajcar, S. Wünsch, M. Siegel, A.N. Omelyanchouk, and O. Astafiev, Phys. Rev. B 81, 172505 (R) (2010).

27. A.N. Omelyanchouk, S.N. Shevchenko, Y.S. Greenberg, O. Astafiev, and E. Il'ichev, Fiz. Nizk. Temp. 36, 1117 (2010) [Low Temp. Phys. 36, 893 (2010)]. 
28. V.I. Shnyrkov, T. Wagner, D. Born, S.N. Shevchenko, W. Krech, A.N. Omelyanchouk, E. Il'ichev, and H.G. Meyer, Phys. Rev. B 73, 024506 (2006).

29. A. Abdumalikov, O. Astafiev, Y. Nakamura, Y. Pashkin, and J. Tsai, Phys. Rev. B 78, 180502(R) (2008).

30. G. Oelsner, Single Artificial-Atom Lasing of a Dressed Flux Qubit, CUVILLIER VERLAG, Göttingen (2017).

31. S.N. Shevchenko, G. Oelsner, Y.S. Greenberg, P. Macha, D.S. Karpov, M. Grajcar, U. Hübner, A.N. Omelyanchouk, and E. Il'ichev, Phys. Rev. B 89, 184504 (2014).

32. A. Izmalkov, S.H.W. van der Ploeg, S.N. Shevchenko, M. Grajcar, E. Il'ichev, U. Hübner, A. N. Omelyanchouk, and H.-G. Meyer, Phys. Rev. Lett. 101, 017003 (2008).

33. M. Jerger, S. Poletto, P. Macha, U. Hübner, A. Lukashenko, E. Il'ichev, and A.V. Ustinov, Europhys. Lett. 96, 40012 (2011).

34. P. Macha, G. Oelsner, J.-M. Reiner, M. Marthaler, S. André, G. Schön, U. Hübner, H.-G. Meyer, E. Il'ichev, and A.V. Ustinov, Nature Commun. 5, 5146 (2014).
35. Y.-F. Chen, D. Hover, S. Sendelbach, L. Maurer, S.T. Merkel, E.J. Pritchett, F.K. Wilhelm, and R. McDermott, Phys. Rev. Lett. 107, 217401 (2011).

36. G. Oelsner, C.K. Andersen, M. Rehák, M. Schmelz, S. Anders, M. Grajcar, U. Hübner, K. Mølmer, and E. Il'ichev, Phys. Rev. Appl. 7, 014012 (2017).

37. M.H. Devoret, J.M. Martinis, and J. Clarke, Phys. Rev. Lett. 55, 1908 (1985).

38. J. Clarke, A.N. Cleland, M.H. Devoret, D. Esteve, and J.M. Martinis, Science 239, 992 (1988).

39. G. Oelsner, L.S. Revin, E. Il'ichev, A.L. Pankratov, H.-G. Meyer, L. Grönberg, J. Hassel, and L.S. Kuzmin, Appl. Phys. Lett. 103, 142605 (2013).

40. C.K. Andersen, G. Oelsner, E. Il'ichev, and K. Mølmer, Phys. Rev. A 89, 033853 (2014). 